Enfermagem Brasil 2016;15(3):137-45

\title{
ARTIGO ORIGINAL \\ Visitando a teoria das transições de Afaf Meleis como suporte teórico para o cuidado de enfermagem
}

\author{
Lanna Gabriela Façanha Costa* \\ *Enfermeira, Mestranda do Programa de Pós-Gradução em Enfermagem da Universidade \\ Federal do Maranhão
}

Recebido em 18 de março de 2015; aceito em 9 de junho de 2015.

Endereço para correspondência: Lanna Gabriela Façanha Costa, Rua 18, quadra 16, casa 11, Planalto Vinhais II, 65074-871 São Luís MA, E-mail: lannagaby@hotmail.com

\section{Resumo}

A teoria das transições proporciona ao enfermeiro auxiliar pessoas a atingirem resultados saudáveis após período de mudança vivenciado. O objetivo do trabalho é oferecer suporte teórico para o cuidado de Enfermagem por meio da teoria das transições. O estudo baseou-se na Teoria das Transições e foi desenvolvido entre os meses de agosto de 2014 e fevereiro de 2015. Diante dos resultados, identificaram-se quatro tipos de transições relevantes para enfermagem: desenvolvimental, situacional, saúde-doença e organizacional. Podem ocorrer simultaneamente, em nível individual, diádico ou familiar, conduzindo a mudança de comportamento e obter comando diante da situação ao encontrar significados e controle. As transições são antecipadas por um acontecimento marcante que requer enfrentamento de estratégias, desenvolvimento de novas competências, funções e relações. Conclui-se que a teoria das transições norteia o enfermeiro a aprofundar-se acerca dos seus conceitos, fazer uso no seu cotidiano na prática do cuidado e planejar ações que favoreçam resultados positivos à saúde.

Palavras-chave: teoria de Enfermagem, cuidados de Enfermagem, Enfermagem.

\section{Abstract \\ Visiting Afaf Meleis' transition theory as theoretical support for nursing care}

The transitions theory provides nurse with a better approach to help people to achieve healthy results after having experienced a period of change. The objective of this work was to provide theoretical support for the nursing care through the transitions theory. The study was based on the transitions theory, developed between August 2014 and February 2015. Based on the results, we identified four types of transitions relevant to nursing: developmental, situational, health-illness and organizational.They may occur simultaneously, either in the individual, dyadic or family level, leading to behavior change and take command of the situation to find meaning and control. Transitions are anticipated by a defining event that requires coping strategies, developing of new skills, roles and relationships. We concluded that the transitions theory guides the nurse to improve his concepts, to use in their daily life in care practices and to plan actions that favor positive health outcomes.

Key-words: Nursing theory, Nursing care, Nursing.

\section{Resumen \\ Visita a la teoría de las transiciones de Afaf Meleis como soporte teórico para el cuidado de enfermería}

La teoría de las transiciones proporciona a los enfermeros la posibilidad de ayudar a las personas a alcanzar resultados saludables después de haber experimentado un periodo de cambio. El objetivo es proveer apoyo teórico para la atención de enfermería a través de la teoría de las transiciones. El estudio se basó en dicha teoria, el cual se desarrolló entre los meses de agosto del 2014 y febrero del 2015. Los resultados permitieron identificar cuatro tipos de transiciones relevantes en el campo de la enfermería: desarrollo, situación, saludenfermedad y organización. Dichas transiciones pueden ocurrir simultáneamente, ya sea a nivel individual, diádico o familiar, lo que lleva a un cambio de comportamiento y al manejo de la situación a fin de encontrar significados y control. Las transiciones pueden ocurrir por un acontecimiento extraordinario que requiere de estrategias de afrontamiento, desarrollo de 
nuevas habilidades, roles y relaciones. Se concluye que la teoría de las transiciones guía a las enfermeras em la profundización de de sus conceptos, ejercitar en la vida cotidiana la práctica de la atención y planificar acciones que favorezcan los resultados positivos para la salud.

Palabras-clave: teoría de Enfermería, cuidados de Enfermería, Enfermería.

Introdução

A teoria das transições exerce forte influência na enfermagem, a partir do desenvolvimento de intervenções que proporcionam um cuidado eficaz anterior a situações de mudanças na vida dos clientes. Entretanto, proporcionar transições saudáveis é uma tarefa desafiante para o enfermeiro, visto que existem pessoas dotadas de singularidades, necessitando prosseguir com novas competências e novas funções nesse processo. Assim, o cuidado de enfermagem se faz necessário com o intuito de proporcionar uma assistência eficiente, melhorando o desfecho dos resultados e evitando transições insalubres [1].

A partir do entendimento da teoria das transições, o enfermeiro deve estabelecer intervenções e estratégias no processo de transição que melhorem a qualidade de vida das pessoas, diminuindo o risco potencial que a experiência de transição pode colocar sobre as pessoas $[1,2]$.

Nesse contexto, a Enfermagem atua de forma holística em todas as dimensões do cuidado, na qual a teoria das transições constitui um meio para orientar seu exercício, permeado pelo contexto de mudanças [3], trazendo como pressuposto a compreensão da transição como processo dinâmico e configurando-se como estratégia para o cuidado de enfermagem. Para isso, descreve-se a Teoria de Afaf Meleis nas suas proposições e etapas, assim como o papel do enfermeiro.

\section{Conceitos de transição}

Transição é uma passagem entre dois períodos de tempo relativamente estáveis, conduzindo o indivíduo a mover-se por diferentes fases dinâmicas, marcos e pontos de mudanças. Esses percursos ocorrem ao longo do tempo e têm um sentido de fluxo e movimento, guiado por alterações que provocam um período de desequilíbrio, incertezas, conflitos interpessoais e perturbações [1,4].

Meleis explica que a transição denota uma mudança no estado de saúde, nas relações de papéis, nas expectativas ou habilidades. Há semelhanças que caracterizam um período de transição: a desconexão com a rede social habitual e sistemas de apoio social; a perda temporária de objetos relevantes ou temas de referência familiar; novas necessidades que possam aparecer e antigos conjuntos de expectativas não mais relativos a situações de mudanças. Sob essa perspectiva, a transição requer que o indivíduo incorpore novos conhecimentos, altere o comportamento, defina seu contexto social, de um ser saudável ou doente, ou das necessidades internas e externas que afeta o estado de saúde [6].

Meleis investigou o que acontece com quem não vivencia transições saudáveis, como os enfermeiros cuidam dessas pessoas, e quais intervenções de enfermagem facilitam o progresso dos clientes no alcance de transições saudáveis. Diante disso, três conceitos foram desenvolvidos: Transições saudáveis como domínio de comportamentos, sentimentos, sinais e símbolos associados com novos papéis; Transições insalubres ou transições ineficazes que movem o indivíduo na direção de vulnerabilidade e risco, tendo a capacidade de resistir às redefinições de significados; Insuficiência do papel caracterizada por dificuldade para desempenhar um papel, em que comportamentos e sentimentos são derivados do descumprimento de obrigações ou expectativas [2].

\section{Material e métodos}

Trata-se de estudo cuja base teórica oferece suporte teórico para o cuidado de Enfermagem por intermédio da Teoria das Transições de Afaf Meleis. A consulta às obras da autora foi iniciada em agosto de 2014, com intuito de embasar a dissertação de Mestrado em Enfermagem da Universidade Federal do Maranhão intulada "O processo de cuidado do recém-nascido prematuro e de baixo peso ao nascer egresso da Unidade de Terapia Intensiva Neonatal em contexto domiciliar". 
Os passos metodológicos foram iniciados a partir da leitura dos referenciais de Afaf Meleis [1-14] e, em seguida, a partir da reflexão e da síntese, descreveram-se os principais fundamentos do constructo teório da Teoria das Transições ao considerar que o mesmo fornece direção suficiente para orientar o cuidado de Enfermagem. Estima-se ser relevante a descrição da teoria e de seus conceitos para compreender seus pressupostos básicos como suporte para os cuidados de Enfermagem e base para sustentar um estudo científico.

Resultados e discussão

\section{O processo de transição para Afaf Meleis}

A Teoria das Transições de Afaf Meleis pode ser objetivamente visualizada na Figura 1, sendo indentificados por meio de um processo colaborativo de entendimento, comparação dos resultados de estudos e análise.

Figura 1 - Diagrama representativo do processo das transições.

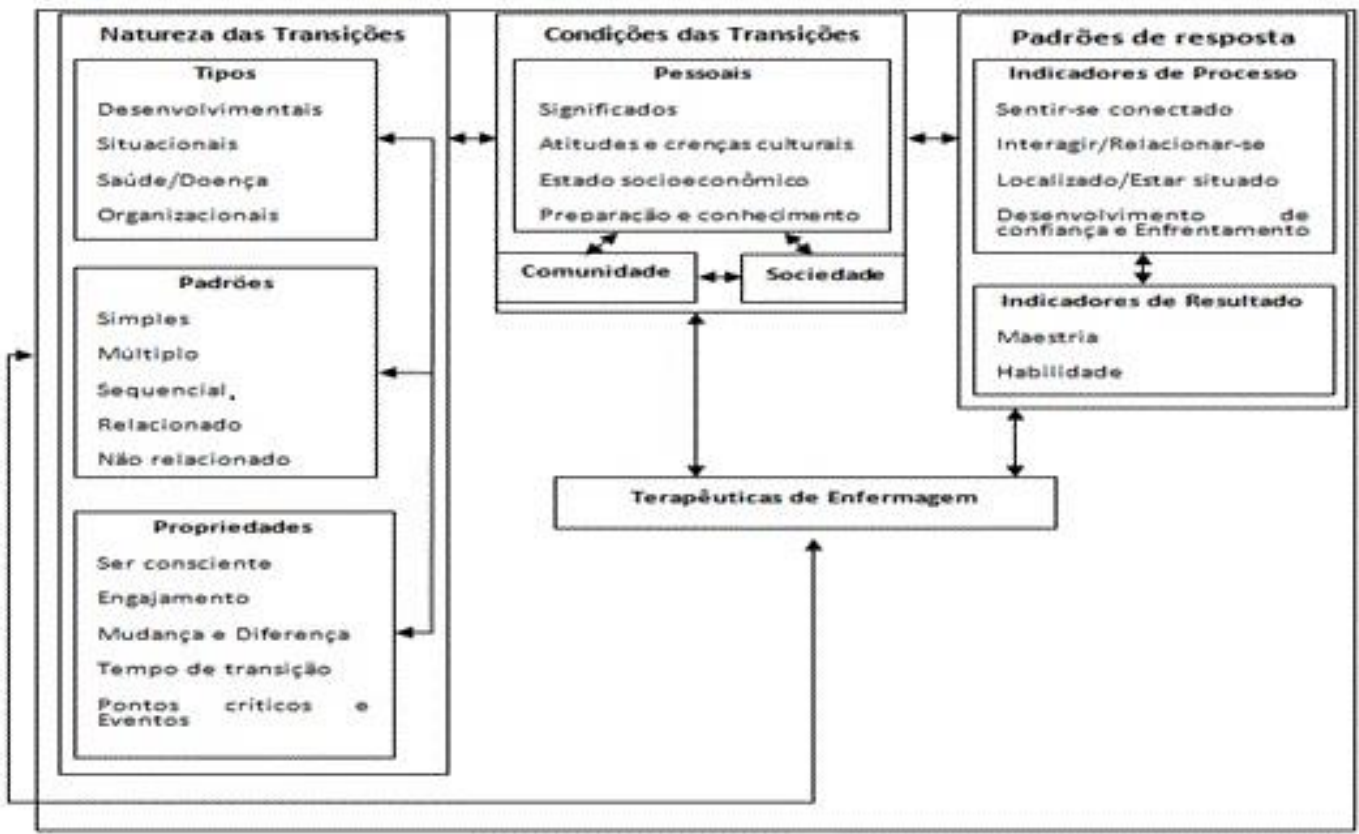

Adaptado de Meleis [1].

\section{Natureza das transições}

A natureza das transições compreende tipos, padrões e propriedades. Schumacher e Meleis [11] identificaram quatro tipos de transições relevantes para a enfermagem: desenvolvimentais, situacionais, saúde-doença e organizacionais. Os tipos de transições não são mutuamente exclusivos, podendo ocorrer simultaneamente durante um determinado período de tempo, em nível individual, didático ou familiar.

Quanto às transições desenvolvimentais, a maior parte dos trabalhos centra-se no indivíduo, compreendendo-a a partir da perspectiva dos que a vivenciam [11], considerando a existência de uma grande variedade de eventos que desencadeiam esse processo de transição, como, por exemplo, relacionamentos de parentalidade (maternidade e paternidade), mudança da imagem corporal na adolescência, menopausa, consciência da homosexualidade e 0 nascimento de uma criança.

As transições situacionais compreendem adição ou subtração de pessoas no meio, requerendo para cada circunstância a definição ou redefinição de papéis dos envolvidos [11]. Mudanças em situações familiares como viuvez, ida do idoso para um asilo, imigração e falta de moradia, caracterizam transições situacionais. Todos estes recebem a atenção dos 
enfermeiros e necessitam de diferentes intervenções de enfermagem e estratégias no processo de transição [2].

A transição no processo saúde/doença é influenciada pela diversidade cultural e de doenças, ficando a passagem do hospital para o domicílio, para o atendimento ambulatorial ou para centros de reabilitação, explorada em vários contextos [1], tais como a ocorrência de doenças cerebrovasculares, recuperação pós-operatória, infecção pelo HIV, lesão da medula espinhal, câncer e doenças crônicas.

Já as Transições Organizacionais representam mudanças em ambientes institucionais e podem ser precipitadas por mudanças no ambiente social, político, econômico,alterações na estrutura ou nas dinâmicas organizacionais [1]. Fazem parte das transições organizacionais: a reorganização estrutural das instalações, a introdução de novos programas, adoção de novas políticas, mudanças em posições de liderança adotando novos padrões de pessoal, implementando novos modelos de cuidados de enfermagem e a introdução de novas tecnologias [11].

Estudos apoiaram também o entendimento das transições como padrões de mutiplicidade e complexidade, nos quais o indivíduo que enfrenta mais de um tipo de transição não a vivencia de maneira isolada e exclusiva, mas em conjunto, podendo um ser prioritário a outro [15]. Diante dos resultados de estudos [14], a natureza das transições sugere que enfermeiros considerem os padrões de todas as transições vivenciadas por um indivíduo, em vez de centrar-se apenas em um tipo específico de transição. Esses padrões podem ser simples ou múltiplos, sequenciais ou simultâneos, de acordo com a dimensão da sobreposição entre as transições e a relação entre os diferentes eventos que precipitam as transições de um indivíduo.

Apesar da complexidade e da multiplicidade das transições, as propriedades das mesmas não são necessariamente exclusivas, mas inter-relacionadas em um processo complexo e dinâmico. Dentre as propriedades [14] incluem ser consciente, engajamento, mudança e diferença, tempo de transição, pontos críticos e eventos.

Ser consciente está associado à percepção, conhecimento e reconhecimento de uma experiência de transição [14] e retrata o conjunto esperado de respostas e percepções dos indivíduos que vivenciaram transições similares e o nível de conformidade entre os processos e as respostas. Chick e Meleis [3] descrevem consciência como uma característica definidora de transição. O indivíduo deverá ter conhecimento das mudanças que estão ocorrendo, uma vez que a ausência desta poderia significar que o indivíduo não iniciou a experiência de transição, apesar da falta de manifestação consciente não ser empecilho do início dessa ocorrência.

O engajamento é o nível de envolvimento de um processo característico à transição. Meleis et al. [14] enfatizam que o nível de consciência influencia o engajamento e este não pode acontecer sem a consciência. O que os autores afirmam é que o nível de envolvimento de um indivíduo que está consciente das mudanças físicas, emocionais, sociais ou ambientais será diferente dos que não vivenciaram tais mudanças.

Mudança e diferença, embora semelhantes, não são sinônimos de transição. Todas as transições envolvem mudança, ao passo que nem todas as mudanças estão relacionadas com a transição [16]. A mudança pode estar relacionada a eventos críticos ou desequilibrador, a perturbações nas relações e rotinas, ou a ideias, percepções e identidades. Deve-se incluir como dimensões da mudança: a natureza, a temporalidade, perceber a importância da gravidade, da pessoa, da família, dos padrões sociais e das expectativas [14].

A diferença é outra propriedade da transição que resulta na alteração de comportamentos ou percepções. Ao analisar as experiências de transição, pode ser útil para que enfermeiros considerem o nível de conforto de um indivíduo e domínio em lidar com a mudança e a diferença [14]. Portanto, essa propriedade compreende alternância nos papéis, na identidade e nos padrões de comportamento, sendo fundamentais para uma visão de si mesmo e do mundo, em vez de mudanças passageiras e superficiais $[3,11,12]$.

Em relação ao tempo de transição, todas as mudanças são caracterizadas por fluxo e movimento ao longo do tempo [12]. Bridges caracteriza transições como um período de tempo com um ponto final identificável, abrangendo desde os primeiros sinais de antecipação, percepção ou demonstração de mudança, até um período de instabilidade, confusão e sofrimento. O tempo de transição envolve o evento inicial marcador até que a harmonia e a estabilidade sejam novamente experimentadas. Este período de tempo é necessário para experimentar diferentes estratégias e padrões de respostas, e, incorporá-los no seu próprio conhecimento $[3,11,12]$. 
Algumas transições são marcadas por acontecimentos, como nascimento, morte ou diagnóstico de uma doença, à medida que, em outras transições, eventos marcadores específicos não são tão perceptíveis [16,17]. Os pontos críticos e eventos são associados com o aumento da conscientização sobre a mudança e diferença, além de engajamento em lidar com a experiência de transição. Há, ainda, pontos críticos, os quais foram caracterizados por um senso de estabilização em novas rotinas, habilidades, estilos de vida e atividades de autocuidado, havendo um período de incerteza marcado com a flutuação, mudanças contínuas e ruptura da realidade. A maioria das experiências de transição envolve pontos críticos e eventos, requerendo do enfermeiro atenção, conhecimento e experiência para lidar com essas mudanças [14].

\section{Condições das transições}

Para entender as experiências do indivíduo durante as transições, é fundamental revelar as condições pessoais e ambientais que facilitam ou dificultam o progresso no sentido de alcançar uma transição saudável. Condições pessoais compreendem os significados, as atitudes e crenças culturais, o estado socioeconômico, a preparação e o conhecimento apoiados nas dimensões comunitárias e sociais, podendo facilitar ou restringir os processos de transições saudáveis e os resultados das transições [14].

Os significados atribuídos a eventos precipitantes de uma mudança podem facilitar ou dificultar transições saudáveis [14]. A maioria das pessoas não relaciona os problemas que teve com a situação vivenciada, indicando que passou pela situação sem sentir ou perceber qualquer adversidade. Assim, "não há significados especiais" para facilitar a transição [18], no entanto significados neutros e positivos podem facilitar a transição.

Atitudes e crenças culturais quando estão ligadas a uma experiência de transição, podem inibir mudanças [14], pois alguns indivíduos se intimidam em discutir sobre algumas situações, preferindo vivenciar a situação por conta própria, tornando-se uma experiência solitária [19]. Seus sintomas psicológicos são observados somente quando há alguma manifestação física [14]. Outro inibidor de transição saudável é a baixa condição socioeconômica. Estudos mostram que indivíduos com baixo nível socioeconômico são mais propensos a apresentar sintomas psicológicos frente a transições [14].

Para Meleis et al. [15], preparar-se antecipadamente para uma mudança facilita a experiência de uma transição, considerando que a falta dessa preparação possa inibir esse percurso. Pode ser útil para gerir a transição a preparação e o conhecimento sobre o que esperar durante a mudança e quais estratégias usarem no processo da transição. As consequências da falta de preparação e compreensão tornam-se imediatamente evidente quando o indivíduo é surpreendido com determinada situação.

Os recursos comunitários, do mesmo modo, facilitam ou inibem as transições. Para lidar com a transição, alguns indivíduos buscam apoio da comunidade diante de situações de saúde e doença, porém os recursos disponíveis nem sempre são aceitos por questões de desconfiança e necessidade de privacidade [18]. As condições que facilitam transições são: 0 apoio da comunidade, informações relevantes obtidas a partir de prestadores de cuidados de saúde confiáveis, conselho de fontes respeitadas, modelos e esclarecimento a perguntas [20]. Inibidores de uma transição saudável incluem: recursos insuficientes para suportar uma situação, suporte inadequado, conselhos não solicitados ou negativos, informação insuficiente ou contraditória, bloqueio por ser estereotipado ou presenciar a negatividade dos outros [20].

No tocante às condições sociais, a sociedade pode facilitar ou inibir as transições. Um evento de transição estigmatizado e com significados estereotipados tendem a interferir no processo de transição saudável. Como exemplo: a desigualdade de gênero como uma restrição no nível da sociedade que influencia a transição e a marginalização da sociedade por conta da própria cultura, sendo negligenciados e ignorados [14].

\section{Padrões de respostas às transições}

Para Schumacher, Jones e Meleis [12], os indicadores de processo de uma transição acontece a qualquer tempo. Temos como exemplo vivenciar sintomas e alteração do estado funcional, conexão com rede interpessoal, recuperação do controle sobre sua vida e senso de integridade englobando o sentido de totalidade e coerência. Para auxiliar nos processos de transição saudável, as terapêuticas de enfermagem favorecem a diminuição das transições insalubres e defende os indicadores de processos positivos. 
Meleis e Trangenstein declaram que "a Enfermagem está preocupada com o processo e as experiências dos seres humanos submetidos às transições, onde a saúde e o bem-estar são o resultado". Ao longo do tempo, as transições se desenvolvem apontando indicadores de processo que movem indivíduos, no sentido de saúde ou vulnerabilidade e risco, o que permite uma avaliação e intervenção precoce facilitando, assim, resultados saudáveis. Esses indicadores incluem: sentir-se conectado, interagir/relacionar-se, estar localizado/situado e desenvolver confiança e enfrentamento [14:257].

A necessidade de sentir-se conectado é ressaltado em muitos relatos de transição [4]. Fazer novos contatos, continuar se conectado com familiares e amigos são exemplos significativos de experiência de um indivíduo [15]. Portanto, utilizar redes sociais e de parentescos são as principais fontes de informações para que os profissionais de saúde identifiquem a emergente dimensão que a transição suporta diante de evidências clínicas $[13,15]$. Outro importante indicador de uma experiência de transição positiva é sentir-se ligado a esses profissionais de saúde que podem esclarecer dúvidas e com quem se pode sentir confortavelmente conectado [15].

Por meio da interação e da relação, comportamentos são desenvolvidos, descobertos, esclarecidos e reconhecidos em resposta à transição [14], e estratégias são desenvolvidas pela interação e reflexão de uma relação nova e emergente. Dessa forma, o envolvimento entre 0 cuidador e o indivíduo cria um contexto de autocuidado e cuidado, ocorrendo de forma eficaz e harmoniosa e recebido como um gesto de apoio [14].

A localização é importante para a maioria das experiências de transição, embora possa ser mais evidente em alguns indivíduos do que em outros [14]. Uma das características de uma transição é a criação de novos sentidos e percepções, quando comparações dão sentido às experiências. Essas comparações são feitas como forma de "situar-se" em relação ao tempo, espaço e relações, tornando uma maneira de explicar e talvez justificar como ou por que indivíduos vão, onde estão, para onde foram e quem são [14].

Diante da dimensão da natureza do processo de transição, existe um padrão indicativo que os indivíduos envolvidos estão experimentando um aumento em seu nível de confiança [14]. Desenvolver a confiança compreende os diferentes processos inerentes ao diagnóstico, tratamento, recuperação e limitações, o nível de utilização de recursos e o desenvolvimento de estratégias. Indivíduos podem demonstrar um conhecimento cumulativo de situações, compreensão de pontos críticos e de mudanças e senso de sabedoria resultante de suas experiências vividas [14].

Indicadores de resultado

À proporção que os resultados são experimentados, refletem diretamente na qualidade de vida daqueles que estão enfrentando transições [14]. A determinação de quando uma transição está completa é variável e flexível, e depende do tipo de alteração ou o evento que inicia a transição, bem como da natureza e o ritmo de transição. Dois indicadores de resultado surgiram a partir de estudos: domínio de novas competências (maestria) e desenvolvimento de uma fluida identidade integrativa (habilidade).

O Papel de Maestria caracteriza o domínio de novos comportamentos exigidos diante de novas situações, domínio de novas competências necessárias para gerir uma transição [14]. Alguns componentes incluindo competência, conhecimento ou habilidade cognitiva, tomada de decisão, habilidades psicomotoras e autoconfiança são características importantes para o papel de maestria. Portanto, a conclusão saudável de uma transição é determinada pelo grau em que indivíduos demonstram domínio das habilidades e comportamentos necessários para gerenciar suas novas situações [7].

As habilidades necessárias para conseguir o controle sob os cuidados de determinada situação incluem o monitoramento e interpretação de sintomas, tomada de decisões, agilidade, fornecimento de cuidado com as mãos e trabalhar em colaboração com o receptor de cuidados [21]. Além disso, a habilidade se desenvolve ao longo do tempo com a experiência, sendo pouco provável ser identificada no início de uma experiência de transição.

\section{O papel da Enfermagem nos processos de transição}

A transição é motivo de apreensão para a enfermagem pelo risco potencial que sua experiência pode colocar sobre as pessoas [1]. Prevenir esses riscos, melhorar o bem-estar, maximizar o funcionamento e reproduzir atividades de autocuidado são estratégias que 
enfermeiros usam para cuidar e apoiar as pessoas a alcançar os processos de transição saudáveis e resultados [1]. Portanto, lidar com a transição é um processo dinâmico, que inclui vários seguimentos, alguns construídos de forma criativa e dinâmica e, ao mesmo tempo, adquirindo experiência e desenvolvendo competências no cuidado [22].

Bridges [16,17] descreve três fases experimentadas na transição, sendo a primeira etapa "um período de terminações" em que há a retirada de relacionamentos ou de formas de comportamento assim como mudanças. A segunda etapa, denominada de "zona neutra", é o período de tempo em que uma pessoa vivencia desorientação causada pelas perdas no primeiro estágio, seguido pela desintegração de sistemas que estavam no local. O estágio final de uma transição é o de "novos começos", marcado por encontrar significado e experimentar controle diante da situação. Segundo o autor, as pessoas devem passar por todos os três estágios para lidar realmente com a transição. No entanto, as fases de uma transição não ocorrem obrigatoriamente de uma forma linear, em vez disso, eles podem ser sequenciais, paralelas ou sobrepostas.

Fundamentadas nos estudos de Schumacher, Jones e Meleis [4], descreve-se a seguir modalidades terapêuticas de enfermagem para lidar com as transições:

Avaliação de Enfermagem é a origem para todas as terapêuticas de enfermagem. Como a transição se modifica, o enfermeiro precisa em uma sequência linear de ações de enfermagem, avaliar, planejar e implementar. Para que os cuidados de enfermagem prosperem, a avaliação deve abranger todo o período de transição, requerendo uma vigilância especial, além de criação de um contexto de cuidados de saúde. A avaliação dos indicadores de processos oferece uma forma de investigar o progresso do indivíduo e detecta precocemente dificuldades em pontos críticos no processo de transição [4,23, 24].

Reminiscência auxilia na integração do processo de transição, fornecendo um elo importante entre o passado e o presente e constrói oportunidades para o indivíduo refletir sobre as experiências de vida. Além disso, facilita o processo de crescimento e desenvolvimento de identidade, facilita os processos de explorar o significado e as áreas nas quais a continuidade com o passado ainda é possível descobrir [25,26].

Suplementação de papel facilita o processo em propagar novos conhecimentos e habilidades. Traz à consciência comportamentos, sentimentos, sensações e objetivos envolvidos em uma determinada função e é particularmente útil para que as pessoas assumam um novo papel [2]. Para Schumacher, Jones e Meleis [4], a suplementação de papel tem vários componentes, entre os quais esclarecer e identificar aspectos, tais como entender a posição e o ponto de vista do outro e entender como o seu papel pode afetar outras pessoas.

Criação de um ambiente saudável há características peculiares dentre as quais Daly e Berman [27], McCracken [23], Taft et al. [28] destacam: fornecer um ambiente que forneça segurança e proteção; respeitar tradições culturais; facilitar o acesso aos que necessitam para realizar rotinas diárias e livrar o meio ambiente de obstáculos. A meta da enfermagem é proporcionar um ambiente dinâmico e adaptável, com o objetivo de sincronizar as necessidades de cada pessoa.

Mobilização de recursos incluem recursos pessoais, familiares e comunitários. Para mobilizar recursos, é necessário considerar sua disponibilidade, se eles são ou não estáveis, se são suficientes ou se é necessário desenvolver novos recursos [4]. As autoras apontam exemplos de estratégias de terapêuticas de enfermagem durante o período de transição, como promoção de estilo de vida saudável, incentivo de exercício regular, uma dieta nutritiva, controle do consumo de álcool, imunizações regulares, além de serviços regulares de cuidados primários de saúde.

Chick e Meleis [3] enfatizam que há variações de transições que ocorrem entre indivíduos, famílias ou organizações. Nesse caso, devem ser avaliadas por enfermeiros para perceberem mudanças e entenderem a experiência de transição de cada indivíduo, assim como a especificidade para compreender os fatores que influenciam o processo de transição, tais como significados, expectativas, nível de conhecimento e habilidade, ambiente, nível de planejamento, nível do bem-estar físico e emocional.

As transições são de notável interesse para a Enfermagem pelas consequências que trazem para a saúde dos clientes. Para evitar instabilidade, enfermeiros precisam levar em consideração padrões de respostas em vez de respostas individuais, reconhecer situações críticas e de vulnerabilidade durante as transições. Diante disso, são indispensáveis as 
terapêuticas de enfermagem com o intuito de impedir consequências negativas e favorecer resultados positivos à saúde. Visitar a teoria das transições proporcionou, além do aprofundamento acerca dos seus conceitos, a utilização no âmbito da vivência cotidiana da prática do cuidado, pretendendo que teorias revelem, sugiram e apoiem o planejamento das ações do profissional de enfermagem, respondendo a suas expectativas e a dos pacientes.

1. Meleis Al. Transitions Theory: middle-range and situation-specific theories in nursing research and practice. New York: Springer; 2010.

2. Meleis Al. Role insufficiency and role supplementation: a conceptual framework. Nurs Res 1975;24:264-71.

3. Chick N, Meleis Al. Transitions: a nursing concern. In: Chinn PL, ed. Nursing research methodology: issues and implementation. Rockville: Aspen; 1986. p. 237-57.

4. Schumacher KL, Jones PS, Meleis AI. Helping elderly persons in transition: a framework for research and practice.In: Swanson EA, Tripp-Reimer T, eds. Life Transitions in the older adult: issues for nurses and other health professionals. New York: Springer; 1999. p. 1-26.

5. Meleis Al. Theory development and domain concepts. In: Moccia P. ed. New approaches to theory development. New York: National League for Nursing; 1986. p. 3 21.

6. Meleis Al. Theoretical nursing: development and progress. 2nd ed. Philadelphia: Lippincott; 1991.

7. Meleis Al, Swendson L. Role supplementation: an empirical test of a nursing intervention. Nurs Res 1978;27(1):11-8.

8. Meleis Al. Women in transition: being versus becoming or being and becoming. Health Care Women Int 1987;8:199-217.

9. Meleis AI, Lipson JG, Paul SM. Ethnicity and health among five middle eastern immigrant groups. Nurs Res 1992;41(2):98-103.

10. Meleis Al. A passion for substance revisited: global transitions and international commitments. St. Paul: Published Keynote Speech given at the 1993 National Doctoral Forum; 1993.

11. Schumacher K, Meleis Al. Transitions: a central concept in nursing. Image J Nurs Sch 1994;26(2):119-27.

12. Meleis Al, Trangenstein PA. Facilitating transitions: redefinition of a nursing mission. Nurs Outlook 1994;42(6):255-9.

13. Lipson JG, Meleis Al. Research with immigrants and refugees. In: Hinshaw A. Feetham S. Shaver J. eds. Hand-book of Clinical Nursing Research. Thousand Oaks: Sage; 1999. p. 87-106.

14. Meleis AI, Sawyer LM, Im EO, Hilfinger Messias DK, Schumacher K. Experiencing transitions: an emerging middle range theory. ANS Adv Nurs Sci 2000;23(1):12-28.

15. Messias DKH. Narratives of Transnational Migration, Work and Health: the lived experiences of Brazilian women in the United States [Doctoral Dissertation]. San Francisco: University of California; 1997.

16. Bridges W. Managing transitions: making the most of change. New York: Da Capo Press; 1991.

17. Bridges W. Transitions. Reading: Addison-Wesley; 1980.

18. Im EO. Neglecting and ignoring menopause within a gendered multiple transitional context: low income Korean immigrant women [Doctoral dissertation]. San Francisco: University of California; 1997.

19. Im EO. An analytical study of the relationship between menopausal symptoms and the stress of life events. J Korea Community Health Nursing Academic Society 1994;8(2):134.

20. Sawyer LM. Engaged mothering within a racist environment: the transition to motherhood for a group of African american women. San Francisco: University of California; 1997.

21. Schumacher KL, Stewart BJ, Archbold PG, Dodd MJ, Dibble SL. Family caregiving skill: development of the concept. Res Nurs Health 2000; 23(3):191-203. 
22. Schumacher KL. Family caregiver role acquisition: role-making through situated interaction. Sch Inq Nurs Pract 1995;9(3):211-29.

23. McCracken AL. Special care units: meeting the needs of cognitively impaires persons. J Gerontol Nurs 1994;20(4):41-46.

24. Moneyham L, Scott CB. Anticipatory coping in the elderly. J Gerontol Nurs 1995;21(7):23-28.

25. Burnside I. Reminiscence: an independent nursing intervention for the elderly. Issues Ment Health Nurs 1990;11:33-48.

26. Burnside I, Haight BK. Reminiscence and life review: analyzing each concept. J Adv Nurs 1992;17:855-62.

27. Daly MP, Berman BM. Rehabilitation in the elderly patient with arthritis. Clin Geriatr Med 1993;9:783-801.

28. Taft LB, Delaney K, Seman D, Stansell J. Creating a therapeuticmilieu in dementia care. J Gerontol Nurs 1993;19(10):30-9. 